\title{
Insights into bacterial protein glycosylation in human microbiota
}

\author{
Fan Zhu \& Hui Wu* \\ Departments of Microbiology and Pediatric Dentistry, Schools of Dentistry and Medicine, University of Alabama at Birmingham, \\ Birmingham, AL 35294, USA
}

Received August 22, 2015; accepted November 5, 2015; published online December 24, 2015

\begin{abstract}
The study of human microbiota is an emerging research topic. The past efforts have mainly centered on studying the composition and genomic landscape of bacterial species within the targeted communities. The interaction between bacteria and hosts is the pivotal event in the initiation and progression of infectious diseases. There is a great need to identify and characterize the molecules that mediate the bacteria-host interaction. Bacterial surface exposed proteins play an important role in the bacteria-host interaction. Numerous surface proteins are glycosylated, and the glycosylation is crucial for their function in mediating the bacterial interaction with hosts. Here we present an overview of surface glycoproteins from bacteria that inhabit three major mucosal environments across human body: oral, gut and skin. We describe the important enzymes involved in the process of protein glycosylation, and discuss how the process impacts the bacteria-host interaction. Emerging molecular details underlying glycosylation of bacterial surface proteins may lead to new opportunities for designing anti-infective small molecules, and developing novel vaccines in order to treat or prevent bacterial infection.
\end{abstract}

microbiota, surface glycoprotein, glycosylation, bacteria-host interaction, glycosyltransferase

Citation: Zhu, F., and Wu, H. (2016). Insights into bacterial protein glycosylation in human microbiota. Sci China Life Sci 59, 11-18. doi: $10.1007 / \mathrm{s} 11427-015-4980-7$

\section{INTRODUCTION}

Protein glycosylation is the covalent linkage of glycans to the amide nitrogen of Asn residues ( $N$-glycosylation) or to the hydroxyl oxygen of Ser or Thr residues (O-glycosylation) (Szymanski and Wren, 2005). As the most abundant post-translational modification in nature, protein glycosylation was once considered to exist only in eukaryotes (Iwashkiw et al., 2013). The protein glycosylation in bacteria and archaea was not appreciated until the findings of such modification from the Halobacterium and Clostridium species 40 years ago (Nothaft and Szymanski, 2010). Since then, the concept of protein glycosylation on bacterial and archaea has been recognized. There are some commonalities and differences in eukaryotic and prokaryotic glycosylation (as reviewed in (Dell et al., 2010)). How-

*Corresponding author (email: hwu@uab.edu) ever, due to the complexity and diversity of the glycosylation systems and the lack of advanced analytical tools of investigation, the rapid growth of studying protein glycosylation in these two kingdoms did not occur until recent decades.

Because of the numerical dominance of bacteria and bacterial genes in human body, a panoply of interests have been ignited to explore this uncharted area. Studying the bacteria-host interaction has led to breakthrough discoveries (Janssen and Kersten, 2015; Lebeer et al., 2010). The key to better understanding the interaction is the identification and characterization of the molecular players present on the bacterial cell surface, often are glycoconjugates such as surface glycosylated proteins (adhesins), exopolysaccharides (EPSs), capsular polysaccharides (CPSs), lipopolysaccharides (LPSs), and peptidoglycan (PG) etc. (Reviewed in (Tytgat and Lebeer, 2014)). In this review, we will focus on one of the glycoconjugates-surface glycoproteins. 
Human mucosal surfaces are associated with abundant bacteria, and the microbiota play a critical role in the physiology and pathology of the host (Consortium, 2012). Of the complex microbiota, both pathogenic and commensal bacteria can establish the initial contacts through their surface proteins (Aviles-Reyes et al., 2014a; Deng et al., 2014; Siboo et al., 2005; Thomer et al., 2014; Yang et al., 2014). These unique bacterial barcodes allow them to gain access to host cells and localize to their specific niches in human, a crucial step for bacteria to maintain their homeostasis with their host. The modification of the bacterial surface proteins by carbohydrates makes the interaction more dynamic (Aviles-Reyes et al., 2014a; Deng et al., 2014; Siboo et al., 2005; Tan et al., 2015; Thomer et al., 2014; Yang et al., 2014). There has been a huge drive for understanding bacterial glycosylation systems in recent decades. Numerous medical important bacterial glycoproteins have been discovered, we will summarize the recent advances of some representative surface glycoproteins in three unique human mucosal surfaces (Figure 1).

\section{SURFACE PROTEIN GLYCOSYLATION IN BACTERIA FOUND IN THE ORAL MUCOSA}

The oral microbial community is one of the most diverse environments in human body. It consists of over 700 different bacterial species and some species of fungi (Jenkinson and Lamont, 2005). Both commensal and pathogenic bacteria reside in the oral cavity (Kreth et al., 2009; Schachtele et al., 2007). These microbes manage to stay in the commensal environment by attaching to host surfaces or to antecedent organisms, which leads to the formation of dental plaque (Zhu et al., 2015b).
Streptococcus parasanguinis, one of the commensal oral streptococci in the oral cavity, utilizes its fimbriae to mediate the initial attachment to tooth surface (Wu et al., 1998). As the major subunit of the fimbriae, fimbriae associated protein 1-Fap1 is the first identified serine-rich repeat glycoproteins (SRRPs) (Froeliger and Fives-Taylor, 2001; Wu and Fives-Taylor, 1999; Zhu et al., 2015b). Glycosylation of Fap1 is critical for bacterial adhesion and biofilm formation (Zhou and Wu, 2009; Zhu et al., 2015b). The glycosylation system of Fap1 has been intensively studied and emerging as a model system to study bacterial protein glycosylation (Bu et al., 2008; Wu et al., 2007a; Wu et al., 2007b; Wu and Wu, 2011; Wu et al., 2010; Zhang et al., 2014; Zhou et al., 2010; Zhu et al., 2011; Zhu et al., 2015a) (Figure 2). We have showed an eleven gene cluster is responsible for the glycosylation and maturation of Fap1. Glycosyltransferases encoded by gtfl and gtf2, and genes from the gly-gtf3galT1-galT2 locus mediate the Fap1 glycosylation (Zhou and Wu, 2009; Zhu et al., 2015b). Fap1 is O-linked with oligosaccharides consisting of GlcNAc, glucose and rhamnose (Zhang et al., 2014). A two-protein enzyme complex Gtf1 and Gtf2 initiates the first step of Fap1 glycosylation by transferring GlcNAc to the serine residues of Fap1 polypeptide backbone (Wu and $\mathrm{Wu}, 2011$ ), and Gtf3 transfers the second carbohydrate-Glc to GlcNAc modified Fap1 (Zhou et al., 2010). A unique domain DUF1975 mediates the third step of Fap1 glycosylation by adding another Glc to GlcNAc-Glc modified Fap1 (Zhang et al., 2014). For the detailed features of each glycosyltransferases, see our recent JDR review (Zhu et al., 2015b). Deletion of each glycosyltransferase in the gene cluster resulted in impaired bacterial biofilm formation and decreased bacterial adherence demonstrating the importance of the Fap1 glycosyla- tion in the bacterial-host interaction.

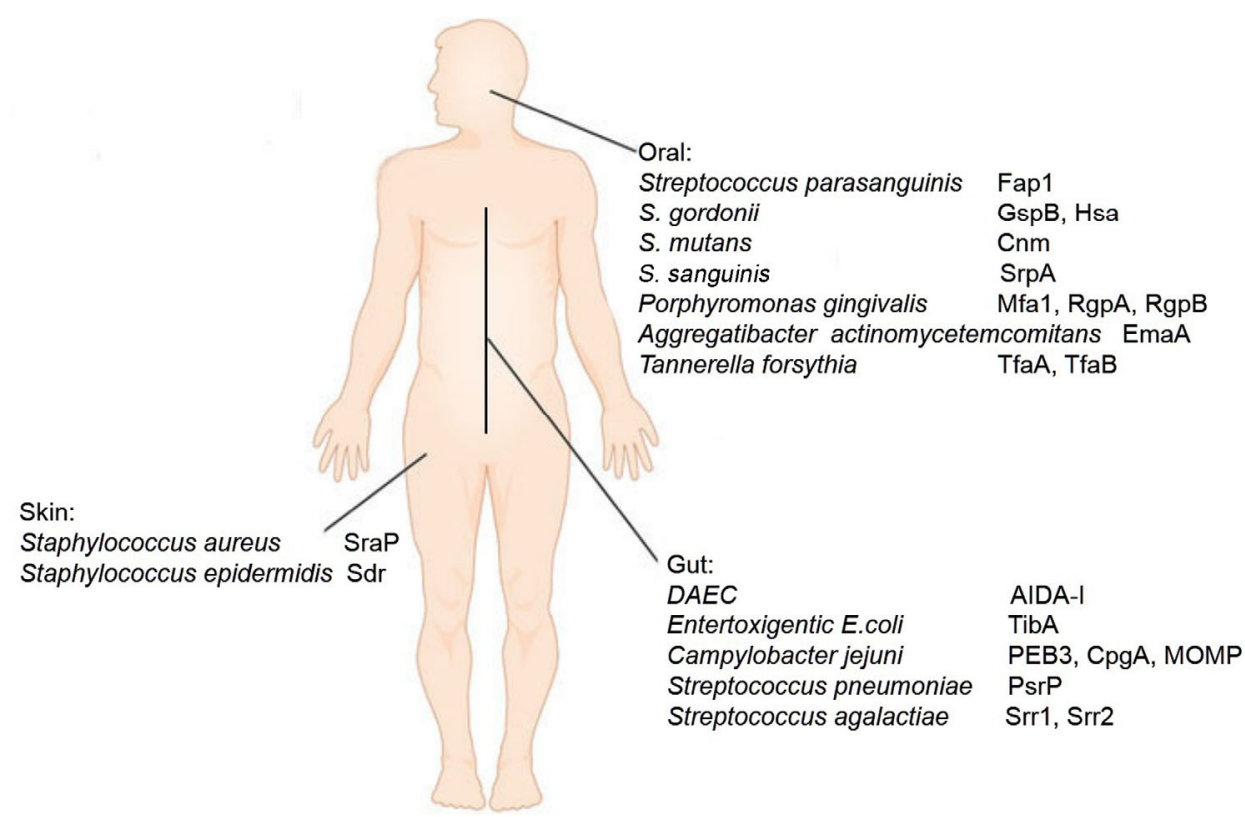

Figure 1 (color online) Microbes and their major host interaction players across human body. 


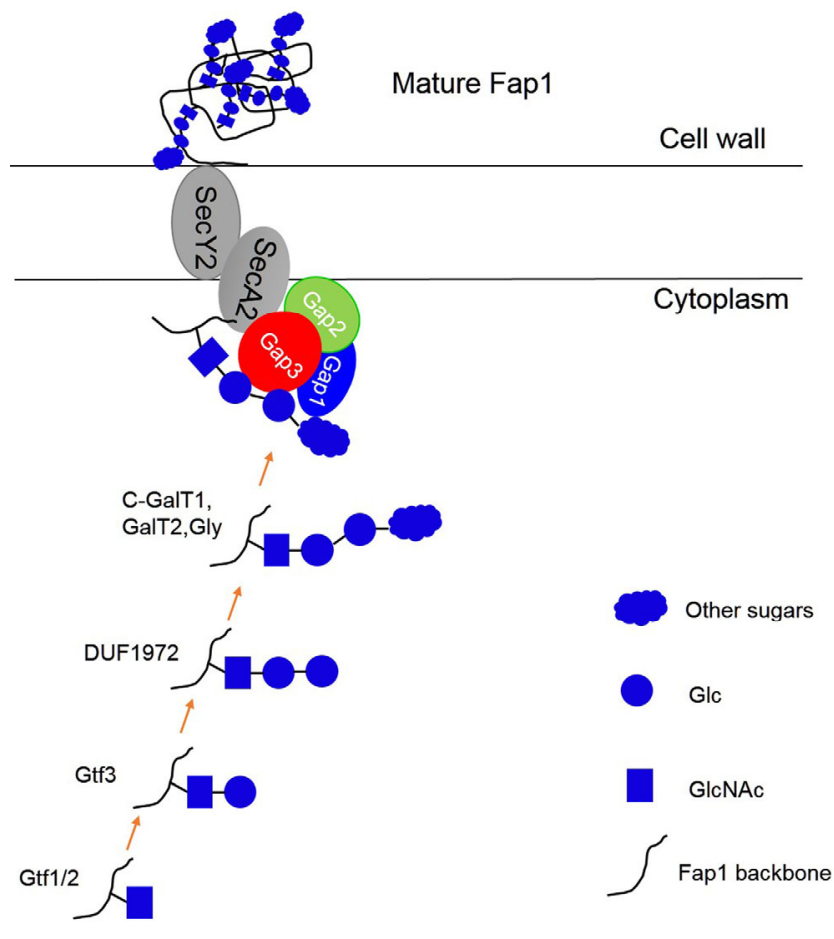

Figure 2 (color online) A proposed model for the biogenesis of SRRP-Fap1 in S. parasanguinis. Fap1 is glycosylated by a series of glycosyltransferases and the modified Fap1 is secreted and anchored to cell wall with the help of a three protein complex (Gap1,2,3) and secretion components SecA2 and SecY2.

Since the discovery of Fap1, two other SRRPs, GspB and Hsa from another commensal streptococci in the oral cavity-Streptococcus gordonii have been identified and characterized. Both GspB and Hsa bind to sialic moieties on the platelets (Bensing et al., 2004) and also to salivary glycoproteins such as MG2, salivary agglutinin and secretory IgA (Heo et al., 2013; Takamatsu et al., 2006) suggesting the SRRP glycoproteins also have lectin activities. Extensive studies were carried out to investigate the GspB glycosylation system. GspB is a SRRP from S. gordonii M99, and the glycosylation of GspB is important for bacterial adhesion to human platelets (Bensing and Sullam, 2002) . The carbohydrate moieties associated with GspB are attached to Ser or Thr residues via an O linkage (Takamatsu et al., 2004a). Four proteins encoded in the gspB-secY2A2 operon of $S$. gordonii mediate the intracellular glycosylation of GspB (Takamatsu et al., 2004a). GtfA and GtfB are the homologous to the Gtf1 and Gtf2, the initiating enzymes of Fap1 glycosylation (Takamatsu et al., 2004a). Gly in the operon is believed to add the terminal sugars to GspB, and nucleotide sugar synthetase (Nss) was thought to be involved in the biosynthesis of UDP-sugar precursors (Takamatsu et al., 2004a). It is worthy nothing that the homologous protein Gtf3 from $S$. parasanguinis was initially also annotated as Nss, by its sequence homology, but biochemical and structural studies unequivocally demonstrated it is a bona fide glucosyltransferase (Zhou et al., 2010; Zhu et al., 2011). Therefore, it is likely Nss from S. gordonii is also a glyco- syltransferase, which needs further studies. Nevertheless, the glycosylation of $\mathrm{GspB}$ enhances the platelet binding activity (Takamatsu et al., 2004b) indicating the importance of these enzymes in mediating the interaction between bacteria and host.

S. mutans, an obligate biofilm forming organism, is an important oral pathogen that mediates the development of dental caries. Biofilm formation is crucial for $S$. mutans virulence. Three glucosyltransferases GtfB, C and GtfD produced by $S$. mutans are responsible for synthesis of biofilm matrix-glucans that promote colonization of the cariogenic bacterium and formation of cariogenic environment (Bowen and Koo, 2011). Interestingly Gtfs all contain glucan binding domains that bind to glucans producing by Gtfs exposed on the bacterial surface, this unique self-promoting adhesion mechanism allows $S$. mutans become a successful cariogenic microbe. Since Gtfs bind to glucans, it has been speculated as glycosylated proteins, but there is no studies demonstrating they are actually modified.

In addition to causing dental caries, S. mutans has been associated with systemic infection. Interestingly one of surface collagen binding protein named Cnm not only contributes to oral colonization but also is linked to bacterial systemic infection as it is capable of invading human coronary artery endothelial cells (Abranches et al., 2011; AvilesReyes et al., 2014b; Miller et al., 2015). Cnm is a glycosylated protein. The glycosylation of $\mathrm{Cnm}$ is controlled by a putative glycosyltransferase PgfS as the deletion of PgfS affects Cnm glycosylation, protein stability and its binding ability to collagen (Aviles-Reyes et al., 2014a). It remains unknown about the carbohydrate composition of $\mathrm{Cnm}$ and whether PgfS is solely responsible for the transfer of sugar residues to the Ser/Thr residues of Cnm.

Another important group of bacteria in the oral cavity are periodontal pathogens. They carry surface glycoproteins that mediate bacterial binding to the host surface. For instance, periodontal pathogens, Porphyromonas gingivalis and Aggregatibacter actinomycetemcomitans possess unique surface glycoproteins to interact with the host.

$P$. gingivalis has two types of cell-surface fimbriae, the major fimbriae known as FimA and the minor fimbriae known as Mfa1 (Zeituni et al., 2010). The major fimbrial protein FimA plays an important role in promoting bacterial attachment to oral surfaces while the minor fimbrial protein Mfa1 is responsible for the microcolony formation and maturation of $P$. gingivalis biofilms (Lin et al., 2006). It is documented that $\mathrm{Mfa} 1$ is a glycoprotein that harbors DC-SIGN-targeting carbohydrates, fucose, mannose, GlcNAc, and GalNAc (Zeituni et al., 2010). The carbohydrate moieties are responsible for $P$. gingivalis interaction with dendritic cells (DCs), which leads to an immunosuppressive DC response (Zeituni et al., 2010). Mfa1 is modified by a mixture of $\mathrm{N}$-glycosylation and $\mathrm{O}$-glycosylation (Zeituni et al., 2010). It remains to be determined how Mfal becomes glycosylated and what machinery engaged. Well known 
virulence factors, gingipains (RpgA and $\operatorname{RgpB}$ ), and outer membrane proteins such as HBP35, OMP85, and Pgm6/7 are also glycosylated on the cell surface of $P$. gingivalis (Curtis et al., 1999; Murakami et al., 2014; Nakao et al., 2008; Shoji et al., 2011). Glycosylation of these proteins is essential for the protein stability, bacterial biofilm formation and the bacterial-host interaction (Hiratsuka et al., 2010; Nakao et al., 2008; Rangarajan et al., 2005). It is known that VimF, a putative glycosyltransferase, mediates glycosylation of gingipains (Vanterpool et al., 2005), however, it is completely unexplored how other proteins are glycosylated. Given the large number of proteins are reported to be glycosylated in $P$. gingivalis, it is reasonable to speculate there is a common glycosylation system in $P$. gingivalis.

A virulence factor, extracellular matrix protein adhesin A (EmaA) from another periodontal pathogen A. actinomycetemcomitans is glycosylated and binds to collagen. The glycosylation of EmaA is modified by enzymes associated with the LPS biosynthetic pathway (Tang and Mintz, 2010; Tang et al., 2012). The shared LPS biogenesis and protein glycosylation pathways are evolutionarily conserved in bacteria (Hug and Feldman, 2011). Sharing biosynthetic pathways are cost effective, which may help enhance bacterial fitness in the host. The glycosylation of EmaA is important for the collagen binding activity and protection of the protein against degradation by proteolytic enzymes (Tang et al., 2012).

Two surface proteins TfsA and TfsB from T. forsythia are also glycosylated (Lee et al., 2006; Posch et al., 2011). But the glycosylation of TfsA and TfsB impacts the life-style of $T$. forsythia very differently. The glycan mutants have increased biofilm formation (Narita et al., 2014). It is possible that the glycosylation of the surface glycoprotein is inhibitory to $T$. forsythia biofilm formation but the underlying mechanism is unclear (Posch et al., 2012). Furthermore, the impaired glycosylation of TfsA and TfsB makes T. forsythia more accessible by dendritic cells (Settem et al., 2013), indicating the importance of the surface glycans in modulating the bacterial interaction with the host immune system.

\section{SURFACE PROTEIN GLYCOSYLATION IN BACTERIA FOUND IN THE GUT MUCOSA ENVIRONMENT}

The human intestinal tract harbors a diverse and complex microbial community which plays a central role in human health and disease. Similar to the oral bacterial colonizers discussed above, bacteria inhabit in the gut also utilize surface structure such as adhesins and flagella to mediate their interaction with host.

There are three glycosylated adhesins reported in E. coli, AIDA-1, TibA and Ag43. The glycosylation of each protein is critical for their function in biofilm formation, autoaggregation, cell adherence and invasion to epithelial cells
(Benz and Schmidt, 2001; Lindenthal and Elsinghorst, 1999; Sherlock et al., 2006). Recent studies suggested AIDA-I is heptosylated within the bacterial cytoplasm by autotransporter adhesin heptosyltransferase (AAH) and its paralogue AAH2, which is defined as a bacterial autotransporter heptosyltransferase (BAHT) family. This family of transferases contains ferric ion and adopts a dodecamer ( $\mathrm{Lu}$ et al., 2014). Structural studies of TibC, a member of BAHT that glycosylates TibA, provide molecular explanation on how the dodecamer enzyme complex manages a high efficient hyperglycosylation of six autotransporter substrates simultaneously (Yao et al., 2014) and shed new insights on bacterial protein glycosylation.

Campylobacter jejuni is the major etiologic agent of bacterial gastroenteritis reported. Flagella is one of the important virulence factors for this organism. Studies have suggested that glycosylation of flagellar is essential for the assembly and motility of flagella in C. jejuni (Alemka et al., 2013). This is also true for other GI tract pathogens such as Helicobacter pylori and Clostridum difficile (Szymanski and Wren, 2005). The major modification on Campylobacter flagellin is O-linked pseudaminic acid (Thibault et al., 2001). Since the glycosylation of flagella has been reviewed recently (Tytgat and Lebeer, 2014), we will not expand on this topic. C. jejuni harbors both O- and N-linked glycosylation system, each has a dedicated gene cluster to catalyze the modification (Day et al., 2012; Guerry and Szymanski, 2008; Szymanski et al., 2003). In addition to flagella, there are a number of surface glycoproteins in $C$. jejuni that play important roles in bacterial attachment to host cells. PEB3 and CgpA are the first identified non-flagellin adhesins that are glycosylated and their glycan components contain $\alpha$ linked N-acetylgalactosamine residues (Linton et al., 2002). It is believed that these surface proteins are N-glycosylated, which may enhance $C$. jejuni fitness by protecting bacterial proteins from cleavage by gut proteases (Alemka et al., 2013). Interestingly, the structure of PEB3 shares high similarity with several periplasmic-binding proteins, indicating PEB3 is a transporter. The exact function of PEB3 remains to be determined (Min et al., 2009; Rangarajan et al., 2007). Recently, the major outer membrane protein MOMP in $C$. jejuni was shown to be O-glycosylated, and the glycosylation is important for binding human histo-blood group antigens and biofilm formation (Mahdavi et al., 2014). It is anticipated that more O-glycosylated surface proteins will be discovered because of advanced analytic glycan detection tools and the presence of a general O-linked glycosylation machinery in $C$. jejuni. Indeed, a large number of non-flagallin O-linked glycoproteins were recently identified in $H$. pylori using glycan metabolic labeling coupled with mass spectrometry analysis and other advanced techniques (Champasa et al., 2013; Hopf et al., 2011).

Streptococcus pneumoniae, the leading cause of otitis media (OM), community-acquired pneumonia, sepsis and meningitis, resides in the upper respiratory tract. It can 
cause pneumonia once it invades the lower respiratory tract. PsrP, a member of SRRPs, is the major virulent factor that mediates the bacterial attachment to Keratin 10 on the surface of lung cells (Sanchez et al., 2010; Shivshankar et al., 2009). This interaction was attributed to the basic region (BR) domain of PsrP that mediated large bacterial aggregates in the nasopharynx and lungs of infected mice via biofilm formation (Schulte et al., 2014). Due to the importance of glycosylation in the function of other SRRPs (Lizcano et al., 2012; Zhu et al., 2015b), one may assume that the glycosylation of PsrP is crucial for bacterial full virulence, but there is no study so far to confirm this hypothesis. Two proteins involved in the glycosylation of PsrP, GtfA and GtfB, have been identified through biochemical and structural studies (Shi et al., 2014; Zhou et al., 2010). GtfA is a glycosyltransferase, other than having a typical catalytic domain, it has a unique domain DUF1975 that has been determined to be an add-on domain. This domain is critical for recognizing the acceptor PsrP (Shi et al., 2014). GtfB enhances the activity of GtfA. It is worth noting that there is no evidence suggesting the binding of PsrP to host cells is lectin dependent, which is different from other characterized SRRPs such as GspB and Hsa. Details regarding how PsrP is fully modified and processed in $S$. pneumoniae is not known and the biological consequences of the modification of PsrP is not clear as well.

Streptococcus agalactiae, also known as Group B Streptococcus, often colonizes GI tract and the vaginal tract. This pathogen emerged in the 1970s as the leading cause of sepsis and meningitis in neonates, but incidences of adult infection also increases recently (http://www.cdc.gov/groupbstrep/clinicians/clinical-overview.html). The major glycosylated bacterial adhesins characterized in two distinct $S$. agalactiae strains are Srr1 and Srr2 (Samen et al., 2007; Seifert et al., 2006; van Sorge et al., 2009), which also are the members of the SRRPs. These two glycoproteins are recognized to mediate bacterial attachment to the host cell surface (Seo et al., 2013; Sheen et al., 2011; Wang et al., 2014). Like the other SRRPs, glycosylation plays a central role in the protein biogenesis and bacterial pathogenesis (Lizcano et al., 2012; Zhu et al., 2015b). Previous studies have shown that GtfA and GtfB catalyze the transfer of GlcNAc to Srr2 polypeptide (Wu et al., 2010). GtfC is important for the second step of glycosylation of Srr2 by catalyzing the direct transfer of glucosyl residues to Srr2-GlcNAc (Zhu et al., 2015a). Structural studies of GtfC identified a conserved loop region that is crucial for acceptor substrate binding from GtfC homologs in streptococci (Zhu et al., 2015a). Mass spectrometry analysis of purified Srr1 unambiguously demonstrates that Serine or Threonine resides on Srr1 was modified by GlcNAc and Glc, which resembles the findings of Srr2 (Chaze et al., 2014). Interestingly there is a novel modification found on Srr1, which was identified as $O$-acetylated- $N$-acetylhex-osamine (Chaze et al., 2014). However, it still remains unknown what en- zyme is responsible for the modification.

\section{SURFACE PROTEIN GLYCOSYLATION IN BACTERIA FOUND IN SKIN FLORA}

More than 1000 species of bacteria habitat upon human skin (Grice et al., 2009). Some of the well-studied bacterial residents include those from the genera Staphylococcus, Corynebacterium, Propionibacterium, Streptococcus and Pseudomonas (Cogen et al., 2008). Staphylococcus epidermidis and Staphylococcus aureus are the most prevalent members of cutaneous microbiota (Chiller et al., 2001; Hazenbos et al., 2013). The infection of host tissues by Staphylococcus requires a family of staphylococcal adhesive proteins that include serine-aspartate dipeptide-repeats (SDR) such as ClfA (clumping factor A) and a number of SDR proteins (Hazenbos et al., 2013). ClfA was recently determined to be modified by GlcNAc (Hazenbos et al., 2013; Thomer et al., 2014). ClfA is critical for capturing fibrin fibrils or fibrinogen and plays an important roles in experimental skin infection (Kwiecinski et al., 2014; Thomer et al., 2014). Two genes coding SdgA and SdgB are involved in the GlcNAc modification of the ClfA SD repeats and other SDR proteins in S. epidermidis and S. aureus (Hazenbos et al., 2013; Thomer et al., 2014). However, only $\mathrm{SdgB}$ is essential for GlcNAc modification. This GlcNAc modification promotes $S$. aureus growth in the bloodstream of mammalian hosts (Thomer et al., 2014).

Because $S$. aureus also can cause human bloodstream infection, sepsis and endocarditis (Yang et al., 2014), many studies have been performed to investigate the bacterial pathogenesis. SraP, a member of SRRPs, mediates the direct binding of $S$. aureus to platelets and its binding to sialylated receptors promotes $S$. aureus adhesion to and invasion into host epithelial cells (Siboo et al., 2005; Yang et al., 2014). GtfA and GtfB, homologs of Gtf1 and Gtf2 from $S$. parasanguinis, are also required for $\mathrm{SraP}$ glycosylation $(\mathrm{Li}$ et al., 2014). The glycosylation pathway is conserved in Gram-positive bacteria found in different body sites.

\section{CONCLUSIONS AND FUTURE PERSPECTIVES}

Bacterial interaction with host cells is a complex and dynamic process involving a variety of bacterial cell surface structures and a host of cell receptors. Many of the surface proteins have evolved to harbor certain carbohydrate modification to facilitate or strengthen their attachment to host cells throughout the human body (Table 1). The modification on these surface glycoproteins might be species or niche specific, but they often share similar glycosylation pathways, either $O$-glycosylation or $\mathrm{N}$-glycosylation.

The emerging studies of bacteria glycoprotein pathways enable to set the stage for the development of glycosylation-based therapeutic strategies, such as glycan-based vaccines to treat or prevent bacterial infection. For instance, 
SRRPs, a growing family of bacterial surface proteins have been demonstrated to be involved in Gram-positive pathogenesis (Lizcano et al., 2012; Zhou and Wu, 2009; Zhu et al., 2015b), which can be explored as novel vaccine candidates. Furthermore, enzymes that are crucial in bacterial $O$-glycosylation but are absent in eukaryotes (Zhang et al., 2014), can be explored as novel antibiotic targets.

Despite the limited understanding of bacterial glycosylation, increasing repertoire of glycosyltransferases identified in the past decade will surely expand the glycoprotein engineering tool box in the context of performing large-scale production of recombinant glycoproteins to generate glycoconjugates with industrial and medical application. Advanced key technologies like MS, NMR, and X-ray crystallography will facilitate characterizing bacterial glycoproteins and the important enzymes involved in the process. In turn, this will undoubtedly stimulate the discovery of new glycosylation pathways and the development of new therapeutic agents for the treatment of bacterial infection and the modulation of microbiota-related diseases.

Compliance and ethics The author(s) declare that they have no conflict of interest.

Acknowledgements The work was supported by NIH (National Institute Of Health)/NIDCR (National Institute of Dental and Craniofacial Research) grants (R01DE017954, R01DE022350).

\section{References}

Abranches, J., Miller, J.H., Martinez, A.R., Simpson-Haidaris, P.J., Burne, R.A., and Lemos, J.A. (2011). The collagen-binding protein Cnm is required for Streptococcus mutans adherence to and intracellular invasion of human coronary artery endothelial cells. Infect Immun 79, 2277-2284.

Alemka, A., Nothaft, H., Zheng, J., and Szymanski, C.M. (2013). $\mathrm{N}$-glycosylation of Campylobacter jejuni surface proteins promotes bacterial fitness. Infect Immun 81, 1674-1682.

Aviles-Reyes, A., Miller, J.H., Simpson-Haidaris, P.J., Hagen, F.K., Abranches, J., and Lemos, J.A. (2014a). Modification of Streptococcus mutans Cnm by PgfS contributes to adhesion, endothelial cell invasion, and virulence. J Bacteriol 196, 2789-2797.

Aviles-Reyes, A., Miller, J.H., Simpson-Haidaris, P.J., Lemos, J.A., and Abranches, J. (2014b). Cnm is a major virulence factor of invasive Streptococcus mutans and part of a conserved three-gene locus. Mol Oral Microbiol 29, 11-23.

Bensing, B.A., Lopez, J.A., and Sullam, P.M. (2004). The Streptococcus gordonii surface proteins GspB and Hsa mediate binding to sialylated carbohydrate epitopes on the platelet membrane glycoprotein Ibalpha. Infect Immun 72, 6528-6537.

Bensing, B.A., and Sullam, P.M. (2002). An accessory sec locus of Streptococcus gordonii is required for export of the surface protein GspB and for normal levels of binding to human platelets. Mol Microbiol 44, 1081-1094.

Benz, I., and Schmidt, M.A. (2001). Glycosylation with heptose residues mediated by the aah gene product is essential for adherence of the AIDA-I adhesin. Mol Microbiol 40, 1403-1413.

Bowen, W.H., and Koo, H. (2011). Biology of Streptococcus mutans-derived glucosyltransferases: role in extracellular matrix formation of cariogenic biofilms. Caries Res 45, 69-86.

Bu, S., Li, Y., Zhou, M., Azadin, P., Zeng, M., Fives-Taylor, P., and Wu,
H. (2008). Interaction between two putative glycosyltransferases is required for glycosylation of a serine-rich streptococcal adhesin. J Bacteriol 190, 1256-1266.

Champasa, K., Longwell, S.A., Eldridge, A.M., Stemmler, E.A., and Dube, D.H. (2013). Targeted identification of glycosylated proteins in the gastric pathogen Helicobacter pylori (Hp). Mol Cell Proteomics 12, 2568-2586.

Chaze, T., Guillot, A., Valot, B., Langella, O., Chamot-Rooke, J., Di Guilmi, A.M., Trieu-Cuot, P., Dramsi, S., and Mistou, M.Y. (2014). $O$-Glycosylation of the N-terminal region of the serine-rich adhesin Srr1 of Streptococcus agalactiae explored by mass spectrometry. Mol Cell Proteomics 13, 2168-2182.

Chiller, K., Selkin, B.A., and Murakawa, G.J. (2001). Skin microflora and bacterial infections of the skin. J Investig Dermatol Symp Proc 6, 170-174.

Cogen, A.L., Nizet, V., and Gallo, R.L. (2008). Skin microbiota: a source of disease or defence? Br J Dermatol 158, 442-455.

Human Microbiome Project Consortium. (2012). Structure, function and diversity of the healthy human microbiome. Nature 486, 207-214.

Curtis, M.A., Thickett, A., Slaney, J.M., Rangarajan, M., Aduse-Opoku, J., Shepherd, P., Paramonov, N., and Hounsell, E.F. (1999). Variable carbohydrate modifications to the catalytic chains of the RgpA and RgpB proteases of Porphyromonas gingivalis W50. Infect Immun 67, 3816-3823.

Day, C.J., Semchenko, E.A., and Korolik, V. (2012). Glycoconjugates play a key role in Campylobacter jejuni infection: interactions between host and pathogen. Front Cell Infect Microbiol 2, 9.

Dell, A., Galadari, A., Sastre, F., and Hitchen, P. (2010). Similarities and differences in the glycosylation mechanisms in prokaryotes and eukaryotes. Int J Microbiol 2010, 148178.

Deng, L., Bensing, B.A., Thamadilok, S., Yu, H., Lau, K., Chen, X., Ruhl, S., Sullam, P.M., and Varki, A. (2014). Oral Streptococci utilize a Siglec-like domain of serine-rich repeat adhesins to preferentially target platelet sialoglycans in human blood. PLoS Pathog 10, e1004540.

Froeliger, E.H., and Fives-Taylor, P. (2001). Streptococcus parasanguis fimbria-associated adhesin fap 1 is required for biofilm formation. Infect Immun 69, 2512-2519.

Grice, E.A., Kong, H.H., Conlan, S., Deming, C.B., Davis, J., Young, A.C., Bouffard, G.G., Blakesley, R.W., Murray, P.R., Green, E.D., Turner, M.L., and Segre, J.A. (2009). Topographical and temporal diversity of the human skin microbiome. Science 324, 1190-1192.

Guerry, P., and Szymanski, C.M. (2008). Campylobacter sugars sticking out. Trends Microbiol 16, 428-435.

Hazenbos, W.L., Kajihara, K.K., Vandlen, R., Morisaki, J.H., Lehar, S.M., Kwakkenbos, M.J., Beaumont, T., Bakker, A.Q., Phung, Q., Swem, L.R., Ramakrishnan, S., Kim, J., Xu, M., Shah, I.M., Diep, B.A., Sai, T., Sebrell, A., Khalfin, Y., Oh, A., Koth, C., Lin, S.J., Lee, B.C., Strandh, M., Koefoed, K., Andersen, P.S., Spits, H., Brown, E.J., Tan, M.W., and Mariathasan, S. (2013). Novel staphylococcal glycosyltransferases $\mathrm{SdgA}$ and $\mathrm{SdgB}$ mediate immunogenicity and protection of virulence-associated cell wall proteins. PLoS Pathog 9, e1003653.

Heo, S.M., Ruhl, S., and Scannapieco, F.A. (2013). Implications of salivary protein binding to commensal and pathogenic bacteria. J Oral Biosci 55, 169-174.

Hiratsuka, K., Kiyama-Kishikawa, M., and Abiko, Y. (2010). Hemin-binding protein 35 (HBP35) plays an important role in bacteria-mammalian cells interactions in Porphyromonas gingivalis. Microb Pathog 48, 116-123.

Hopf, P.S., Ford, R.S., Zebian, N., Merkx-Jacques, A., Vijayakumar, S., Ratnayake, D., Hayworth, J., and Creuzenet, C. (2011). Protein glycosylation in Helicobacter pylori: beyond the flagellins? PLoS One 6, e25722.

Hug, I., and Feldman, M.F. (2011). Analogies and homologies in lipopolysaccharide and glycoprotein biosynthesis in bacteria. Glycobiology 21, $138-151$.

Iwashkiw, J.A., Vozza, N.F., Kinsella, R.L., and Feldman, M.F. (2013). Pour some sugar on it: the expanding world of bacterial protein O-linked glycosylation. Mol Microbiol 89, 14-28.

Janssen, A.W., and Kersten, S. (2015). The role of the gut microbiota in 
metabolic health. FASEB J 29, 3111-3123.

Jenkinson, H.F., and Lamont, R.J. (2005). Oral microbial communities in sickness and in health. Trends Microbiol 13, 589-595.

Kreth, J., Merritt, J., and Qi, F. (2009). Bacterial and host interactions of oral Streptococci. DNA Cell Biol 28, 397-403.

Kwiecinski, J., Jin, T., and Josefsson, E. (2014). Surface proteins of Staphylococcus aureus play an important role in experimental skin infection. APMIS 122, 1240-1250.

Lebeer, S., Vanderleyden, J., and De Keersmaecker, S.C. (2010). Host interactions of probiotic bacterial surface molecules: comparison with commensals and pathogens. Nat Rev Microbiol 8, 171-184.

Lee, S.W., Sabet, M., Um, H.S., Yang, J., Kim, H.C., and Zhu, W. (2006). Identification and characterization of the genes encoding a unique surface (S-) layer of Tannerella forsythia. Gene 371, 102-111.

Li, Y., Huang, X., Li, J., Zeng, J., Zhu, F., Fan, W., and Hu, L. (2014). Both GtfA and GtfB are required for SraP glycosylation in Staphylococcus aureus. Curr Microbiol 69, 121-126.

Lin, X., Wu, J., and Xie, H. (2006). Porphyromonas gingivalis minor fimbriae are required for cell-cell interactions. Infect Immun 74 , 6011-6015.

Lindenthal, C., and Elsinghorst, E.A. (1999). Identification of a glycoprotein produced by enterotoxigenic Escherichia coli. Infect Immun 67, 4084-4091.

Linton, D., Allan, E., Karlyshev, A.V., Cronshaw, A.D., and Wren, B.W. (2002). Identification of $\mathrm{N}$-acetylgalactosamine-containing glycoproteins PEB3 and CgpA in Campylobacter jejuni. Mol Microbiol 43, 497-508.

Lizcano, A., Sanchez, C.J., and Orihuela, C.J. (2012). A role for glycosylated serine-rich repeat proteins in gram-positive bacterial pathogenesis. Mol Oral Microbiol 27, 257-269.

Lu, Q., Yao, Q., Xu, Y., Li, L., Li, S., Liu, Y., Gao, W., Niu, M., Sharon, M., Ben-Nissan, G., Zamyatina, A., Liu, X., Chen, S., and Shao, F. (2014). An iron-containing dodecameric heptosyltransferase family modifies bacterial autotransporters in pathogenesis. Cell Host Microbe $16,351-363$

Mahdavi, J., Pirinccioglu, N., Oldfield, N.J., Carlsohn, E., Stoof, J., Aslam, A., Self, T., Cawthraw, S.A., Petrovska, L., Colborne, N., Sihlbom, C., Boren, T., Wooldridge, K.G., and Ala'Aldeen, D.A. (2014). A novel O-linked glycan modulates Campylobacter jejuni major outer membrane protein-mediated adhesion to human histo-blood group antigens and chicken colonization. Open Biol 4, 130202.

Miller, J.H., Aviles-Reyes, A., Scott-Anne, K., Gregoire, S., Watson, G.E., Sampson, E., Progulske-Fox, A., Koo, H., Bowen, W.H., Lemos, J.A., and Abranches, J. (2015). The collagen binding protein Cnm contributes to oral colonization and cariogenicity of Streptococcus mutans OMZ175. Infect Immun 83, 2001-2010.

Min, T., Vedadi, M., Watson, D.C., Wasney, G.A., Munger, C., Cygler, M., Matte, A., and Young, N.M. (2009). Specificity of Campylobacter jejuni adhesin PEB3 for phosphates and structural differences among its ligand complexes. Biochemistry 48, 3057-3067.

Murakami, Y., Hasegawa, Y., Nagano, K., and Yoshimura, F. (2014). Characterization of wheat germ agglutinin lectin-reactive glycosylated OmpA-like proteins derived from Porphyromonas gingivalis. Infect Immun 82, 4563-4571.

Nakao, R., Tashiro, Y., Nomura, N., Kosono, S., Ochiai, K., Yonezawa, H., Watanabe, H., and Senpuku, H. (2008). Glycosylation of the OMP85 homolog of Porphyromonas gingivalis and its involvement in biofilm formation. Biochem Biophys Res Commun 365, 784-789.

Narita, Y., Sato, K., Yukitake, H., Shoji, M., Nakane, D., Nagano, K., Yoshimura, F., Naito, M., and Nakayama, K. (2014). Lack of a surface layer in Tannerella forsythia mutants deficient in the type IX secretion system. Microbiology 160, 2295-2303.

Nothaft, H., and Szymanski, C.M. (2010). Protein glycosylation in bacteria: sweeter than ever. Nat Rev Microbiol 8, 765-778.

Posch, G., Pabst, M., Brecker, L., Altmann, F., Messner, P., and Schaffer, C. (2011). Characterization and scope of S-layer protein O-glycosylation in Tannerella forsythia. J Biol Chem 286, 38714-38724.

Posch, G., Sekot, G., Friedrich, V., Megson, Z.A., Koerdt, A., Messner, P., and Schaffer, C. (2012). Glycobiology aspects of the periodontal pathogen Tannerella forsythia. Biomolecules 2, 467-482.

Rangarajan, E.S., Bhatia, S., Watson, D.C., Munger, C., Cygler, M., Matte, A., and Young, N.M. (2007). Structural context for protein $\mathrm{N}$-glycosylation in bacteria: the structure of PEB3, an adhesin from Campylobacter jejuni. Protein Sci 16, 990-995.

Rangarajan, M., Hashim, A., Aduse-Opoku, J., Paramonov, N., Hounsell, E.F., and Curtis, M.A. (2005). Expression of Arg-Gingipain RgpB is required for correct glycosylation and stability of monomeric Arg-gingipain RgpA from Porphyromonas gingivalis W50. Infect Immun 73, 4864-4878.

Samen, U., Eikmanns, B.J., Reinscheid, D.J., and Borges, F. (2007). The surface protein Srr-1 of Streptococcus agalactiae binds human keratin 4 and promotes adherence to epithelial HEp-2 cells. Infect Immun 75, 5405-5414.

Sanchez, C.J., Shivshankar, P., Stol, K., Trakhtenbroit, S., Sullam, P.M., Sauer, K., Hermans, P.W., and Orihuela, C.J. (2010). The pneumococcal serine-rich repeat protein is an intra-species bacterial adhesin that promotes bacterial aggregation in vivo and in biofilms. PLoS Pathog 6, e1001044.

Schachtele, C.F., Nobbs, A.H., Zhang, Y., Costalonga, M., and Herzberg, M.C. (2007). Oral Streptococci: commensals and opportunistic pathogens. In: Molecular Biology of Streptococci. R.M. Hakenback, S. Chhatwal, eds. (Norfolk: Horizon Bioscience), pp. 461-462.

Schulte, T., Lofling, J., Mikaelsson, C., Kikhney, A., Hentrich, K., Diamante, A., Ebel, C., Normark, S., Svergun, D., Henriques-Normark, B., and Achour, A. (2014). The basic keratin 10-binding domain of the virulence-associated pneumococcal serine-rich protein PsrP adopts a novel MSCRAMM fold. Open Biol 4, 130090.

Seifert, K.N., Adderson, E.E., Whiting, A.A., Bohnsack, J.F., Crowley, P.J., and Brady, L.J. (2006). A unique serine-rich repeat protein (Srr-2) and novel surface antigen (epsilon) associated with a virulent lineage of serotype III Streptococcus agalactiae. Microbiology 152, 1029-1040.

Seo, H.S., Xiong, Y.Q., and Sullam, P.M. (2013). Role of the serine-rich surface glycoprotein Srr1 of Streptococcus agalactiae in the pathogenesis of infective endocarditis. PLoS One 8, e64204.

Settem, R.P., Honma, K., Nakajima, T., Phansopa, C., Roy, S., Stafford, G.P., and Sharma, A. (2013). A bacterial glycan core linked to surface (S)-layer proteins modulates host immunity through Th17 suppression. Mucosal Immunol 6, 415-426.

Sheen, T.R., Jimenez, A., Wang, N.Y., Banerjee, A., van Sorge, N.M., and Doran, K.S. (2011). Serine-rich repeat proteins and pili promote Streptococcus agalactiae colonization of the vaginal tract. J Bacteriol 193, 6834-6842.

Sherlock, O., Dobrindt, U., Jensen, J.B., Munk Vejborg, R., and Klemm, P. (2006). Glycosylation of the self-recognizing Escherichia coli Ag43 autotransporter protein. J Bacteriol 188, 1798-1807.

Shi, W.W., Jiang, Y.L., Zhu, F., Yang, Y.H., Shao, Q.Y., Yang, H.B., Ren, Y.M., Wu, H., Chen, Y., and Zhou, C.Z. (2014). Structure of a novel O-linked N-acetyl-D-glucosamine (O-GlcNAc) transferase, GtfA, reveals insights into the glycosylation of pneumococcal serine-rich repeat adhesins. J Biol Chem 289, 20898-20907.

Shivshankar, P., Sanchez, C., Rose, L.F., and Orihuela, C.J. (2009). The Streptococcus pneumoniae adhesin PsrP binds to Keratin 10 on lung cells. Mol Microbiol 73, 663-679.

Shoji, M., Sato, K., Yukitake, H., Kondo, Y., Narita, Y., Kadowaki, T., Naito, M., and Nakayama, K. (2011). Por secretion system-dependent secretion and glycosylation of Porphyromonas gingivalis hemin-binding protein 35. PLoS One 6, e21372.

Siboo, I.R., Chambers, H.F., and Sullam, P.M. (2005). Role of SraP, a serine-rich surface protein of Staphylococcus aureus, in binding to human platelets. Infect Immun 73, 2273-2280.

Szymanski, C.M., Logan, S.M., Linton, D., and Wren, B.W. (2003). Campylobacter-a tale of two protein glycosylation systems. Trends Microbiol 11, 233-238.

Szymanski, C.M., and Wren, B.W. (2005). Protein glycosylation in bacterial mucosal pathogens. Nat Rev Microbiol 3, 225-237.

Takamatsu, D., Bensing, B.A., Prakobphol, A., Fisher, S.J., and Sullam, P.M. (2006). Binding of the streptococcal surface glycoproteins GspB 
and Hsa to human salivary proteins. Infect Immun 74, 1933-1940.

Takamatsu, D., Bensing, B.A., and Sullam, P.M. (2004a). Four proteins encoded in the gspB-secY2A2 operon of Streptococcus gordonii mediate the intracellular glycosylation of the platelet-binding protein GspB. J Bacteriol 186, 7100-7111.

Takamatsu, D., Bensing, B.A., and Sullam, P.M. (2004b). Genes in the accessory sec locus of Streptococcus gordonii have three functionally distinct effects on the expression of the platelet-binding protein GspB. Mol Microbiol 52, 189-203.

Tan, F.Y., Tang, C.M., and Exley, R.M. (2015). Sugar coating: bacterial protein glycosylation and host-microbe interactions. Trends Biochem Sci 40, 342-350

Tang, G., and Mintz, K.P. (2010). Glycosylation of the collagen adhesin EmaA of Aggregatibacter actinomycetemcomitans is dependent upon the lipopolysaccharide biosynthetic pathway. J Bacteriol 192, 1395-1404.

Tang, G., Ruiz, T., and Mintz, K.P. (2012). O-polysaccharide glycosylation is required for stability and function of the collagen adhesin EmaA of Aggregatibacter actinomycetemcomitans. Infect Immun 80, 28682877.

Thibault, P., Logan, S.M., Kelly, J.F., Brisson, J.R., Ewing, C.P., Trust, T.J., and Guerry, P. (2001). Identification of the carbohydrate moieties and glycosylation motifs in Campylobacter jejuni flagellin. J Biol Chem 276, 34862-34870.

Thomer, L., Becker, S., Emolo, C., Quach, A., Kim, H.K., Rauch, S., Anderson, M., Leblanc, J.F., Schneewind, O., Faull, K.F., and Missiakas, D. (2014). N-acetylglucosaminylation of serine-aspartate repeat proteins promotes Staphylococcus aureus bloodstream infection. J Biol Chem 289, 3478-3486.

Tytgat, H.L., and Lebeer, S. (2014). The sweet tooth of bacteria: common themes in bacterial glycoconjugates. Microbiol Mol Biol 78, 372-417.

van Sorge, N.M., Quach, D., Gurney, M.A., Sullam, P.M., Nizet, V., and Doran, K.S. (2009). The group B streptococcal serine-rich repeat 1 glycoprotein mediates penetration of the blood-brain barrier. J Infect Dis 199, 1479-1487.

Vanterpool, E., Roy, F., and Fletcher, H.M. (2005). Inactivation of vimF, a putative glycosyltransferase gene downstream of vimE, alters glycosylation and activation of the gingipains in Porphyromonas gingivalis W83. Infect Immun 73, 3971-3982.

Wang, N.Y., Patras, K.A., Seo, H.S., Cavaco, C.K., Rosler, B., Neely, M.N., Sullam, P.M., and Doran, K.S. (2014). Group B streptococcal serine-rich repeat proteins promote interaction with fibrinogen and vaginal colonization. J Infect Dis 210, 982-991.

Wu, H., Bu, S., Newell, P., Chen, Q., and Fives-Taylor, P. (2007a). Two gene determinants are differentially involved in the biogenesis of Fap1 precursors in Streptococcus parasanguis. J Bacteriol 189, 1390-1398.

Wu, H., and Fives-Taylor, P.M. (1999). Identification of dipeptide repeats and a cell wall sorting signal in the fimbriae-associated adhesin, Fap1, of Streptococcus parasanguis. Mol Microbiol 34, 1070-1081.

Wu, H., Mintz, K.P., Ladha, M., and Fives-Taylor, P.M. (1998). Isolation and characterization of Fap1, a fimbriae-associated adhesin of Streptococcus parasanguis FW213. Mol Microbiol 28, 487-500.

Wu, H., Zeng, M., and Fives-Taylor, P. (2007b). The glycan moieties and the N-terminal polypeptide backbone of a fimbria-associated adhesin, Fap1, play distinct roles in the biofilm development of Streptococcus parasanguinis. Infect Immun 75, 2181-2188.

$\mathrm{Wu}, \mathrm{R}$., and $\mathrm{Wu}, \mathrm{H}$. (2011). A molecular chaperone mediates a two-protein enzyme complex and glycosylation of serine-rich streptococcal adhesins. J Biol Chem 286, 34923-34931.

Wu, R., Zhou, M., and Wu, H. (2010). Purification and characterization of an active $\mathrm{N}$-acetylglucosaminyltransferase enzyme complex from Streptococci. Appl Environ Microbiol 76, 7966-7971.

Yang, Y.H., Jiang, Y.L., Zhang, J., Wang, L., Bai, X.H., Zhang, S.J., Ren, Y.M., Li, N., Zhang, Y.H., Zhang, Z., Gong, Q., Mei, Y., Xue, T., Zhang, J.R., Chen, Y., and Zhou, C.Z. (2014). Structural insights into-* SraP-mediated Staphylococcus aureus adhesion to host cells. PLoS Pathog 10, e1004169.

Yao, Q., Lu, Q., Wan, X., Song, F., Xu, Y., Hu, M., Zamyatina, A., Liu, X., Huang, N., Zhu, P., and Shao, F. (2014). A structural mechanism for bacterial autotransporter glycosylation by a dodecameric heptosyltransferase family. Elife doi: 10.7554/eLife.03714.

Zeituni, A.E., McCaig, W., Scisci, E., Thanassi, D.G., and Cutler, C.W. (2010). The native 67-kilodalton minor fimbria of Porphyromonas gingivalis is a novel glycoprotein with DC-SIGN-targeting motifs. J Bacteriol 192, 4103-4110.

Zhang, H., Zhu, F., Yang, T., Ding, L., Zhou, M., Li, J., Haslam, S.M., Dell, A., Erlandsen, H., and Wu, H. (2014). The highly conserved domain of unknown function 1792 has a distinct glycosyltransferase fold. Nat Commun 5, 4339.

Zhou, M., and Wu, H. (2009). Glycosylation and biogenesis of a family of serine-rich bacterial adhesins. Microbiology 155, 317-327.

Zhou, M., Zhu, F., Dong, S., Pritchard, D.G., and Wu, H. (2010). A novel glucosyltransferase is required for glycosylation of a serine-rich adhesin and biofilm formation by Streptococcus parasanguinis. J Biol Chem $285,12140-12148$.

Zhu, F., Erlandsen, H., Ding, L., Li, J., Huang, Y., Zhou, M., Liang, X., Ma, J., and Wu, H. (2011). Structural and functional analysis of a new subfamily of glycosyltransferases required for glycosylation of serine-rich streptococcal adhesins. J Biol Chem 286, 27048-27057.

Zhu, F., Zhang, H., and Wu, H. (2015a). A conserved domain is crucial for acceptor substrate binding in a family of glucosyltransferases. J Bacteriol 197, 510-517.

Zhu, F., Zhang, H., and Wu, H. (2015b). Glycosyltransferase-mediated sweet modification in oral Streptococci. J Dent Res 94, 659-665.

Open Access This article is distributed under the terms of the Creative Commons Attribution License which permits any use, distribution, and reproduction in any medium, provided the original author(s) and source are credited. 